\title{
ANÁLISIS COMPARATIVO DE NÚCLEOS PROCEDENTES DEL EXTREMO SUR DE PATAGONIA CONTINENTAL (REP. ARGENTINA): MATERIAS PRIMAS Y TÉCNICAS DE REDUCCIÓN
}

\author{
JUDITH CHARLIN $^{1}$ Y MARCELO CARDILLO $^{2}$
}

\begin{abstract}
RESUMEN
El objetivo central de este trabajo es el estudio de las estrategias de utilización de las materias primas líticas en el sector meridional de la Provincia de Santa Cruz, República Argentina. Se comparan muestras de núcleos recuperados en diversas localidades dentro del campo volcánico Pali Aike y de la zona costera de Cañadón Gap. Con el fin de evaluar posibles diferencias y similitudes entre los conjuntos se realizaron análisis tanto cualitativos como cuantitativos. Los resultados obtenidos sugieren homogeneidad en las estrategias de explotación de los recursos líticos tanto en la zona costera como en el interior. Los datos obtenidos se discuten en el marco de la información ya existente para el área, en términos de rango de acción de las poblaciones humanas.
\end{abstract}

PALABRAS CLAVES: núcleos, materias primas líticas, diversidad, estrategias de explotación.

\section{COMPARATIVE ANALYSIS OF LITHIC CORES FROM SOUTHERN PATAGONIA: RAW MATERIALS AND REDUCTION TECHNIQUES}

\begin{abstract}
The main goal of this contribution is to study the use strategies of lithic raw material in the southern portion of the Santa Cruz province, Argentina. We compare samples from lithic cores collected at a variety of locales in the Pali Aike Lava Field and in the coastal area of Cañadón Gap. In order to evaluate possible differences and similarities, we use both qualitative and quantitative analysis. Our results show similarity in coastal and hinterland strategies of lithic raw material exploitation. We evaluate this information in relation to other lines of regional evidence, and consider its implications for discussions of the home ranges of human populations.
\end{abstract}

KEY WORDS: Lithic cores, lithic raw materials, diversity, exploitation strategies.

1 Agencia Nacional de Promoción Científica y Técnica. DIPA-IMHICIHU-CONICET, Saavedra 15 5ํㅜ piso Cap. Fed., Rep. Argentina. E-mail: jucharlin@yahoo.com.ar

2 DIPA-IMHICIHU-CONICET, Saavedra 15 5ํ piso Cap. Fed., Rep. Argentina. E-mail: marcelo_cardillo@hotmail.com 


\section{INTRODUCCIÓN}

El presente trabajo se inserta dentro de un proyecto mayor ${ }^{1}$, cuyo objetivo general es el estudio de los modos de interacción entre las poblaciones humanas de Patagonia Meridional (Borrero 2001). En la discusión de esta problemática, la distribución de los artefactos líticos y de las materias primas utilizadas en su manufactura son considerados indicadores de interacción o rangos de acción (Borrero op. cit.). A través de su estudio se intenta distinguir la importancia relativa del intercambio y el abastecimiento directo. Los resultados que aquí se presentan intentan contribuir en esta discusión para el sector meridional de la provincia de Santa Cruz, Argentina.

El objetivo particular de este trabajo es caracterizar el modo de utilización de las materias primas líticas al sur del río Gallegos, a partir del análisis comparativo de los núcleos recuperados en dos sectores de este espacio: el cañadón Gap en la franja próxima a la línea de costa y el campo volcánico Pali Aike localizado hacia el interior.

Esta información es puesta en relación con la disponibilidad de materias primas líticas en estos espacios.

\section{GEOLOGÍA Y GEOMORFOLOGÍA DEL ÁREA DE ESTUDIO}

El campo volcánico Pali Aike se localiza en la provincia estructural conocida como cuenca Austral o de Magallanes, la cual se dispone al este de los Andes entre los $46^{\circ}$ y $55^{\circ} \mathrm{S}$ (Skewes 1978; Agostini et al. 1999; Corbella 2002; D'Orazio et al. 2000). Considerando los límites nacionales y provinciales, la mayor parte del campo volcánico Pali Aike se encuentra en territorio argentino, en el sector meridional de la Pcia. de Santa Cruz, entre el río Coig o Coyle $\left(50^{\circ} \mathrm{S}\right)$ y la frontera con Chile, extendiéndose -ya en este país- hasta el estrecho de Magallanes (52 ${ }^{\circ} \mathrm{S}$ ) (Corbella op. cit.). El mismo cubre un área de $4.500 \mathrm{~km}^{2}$ (D'Orazio et. al op. cit.), extendiéndose hasta aproximadamente $20 \mathrm{~km}$ de la costa atlántica.

1 "Modos de interacción entre las poblaciones humanas de la Patagonia Meridional” (PICT № 04-9498), dirigido por el Dr. L. A. Borrero.
La actividad volcánica que dio origen a la formación del campo Pali Aike comenzó en el Plioceno Tardío y continuó episódicamente hasta el Holoceno (Corbella op. cit.; D'Orazio et al. op. cit). Según los análisis petrográficos y geoquímicos realizados las lavas que conforman el campo son basaltos alcalinos. Considerando los distintos momentos de formación, es posible distinguir tres unidades volcánicas, que varían en cuanto a su localización espacial, las características geomorfológicas que presentan y su composición relativa en elementos mayoritarios (Skewes op. cit.; Agostini et al. op. cit.; Corbella op. cit.; D'Orazio et al. op. cit.). Dichas unidades se asientan sobre sedimentos glaciares, niveles de terrazas glacifluviales pliocenas y pleistocenas, generalmente cubiertas por una capa de "Rodados Patagónicos" o directamente sobre la formación Santa Cruz (Corbella op. cit.).

El área de cañadón Gap se localiza a 60 $\mathrm{km}$ aproximadamente del límite meridional del campo volcánico, siendo la distancia máxima con respecto al sector norte de Pali Aike de $90 \mathrm{~km}$. Este cañadón desemboca en la costa atlántica a unos $25 \mathrm{~km}$ del sitio Cabo Vírgenes 6, entre los $52^{\circ} 08^{\prime} 26.7^{\prime \prime}$ Sur y $68^{\circ} 33^{\prime} 01.0^{\prime \prime}$ Oeste. Se extiende perpendicularmente a la costa atlántica, terminando en una playa de erosión limitada por acantilados. Los cañadones constituyen, por lo tanto, los únicos accesos a la costa y a sus recursos en este sector del espacio. Tanto en la costa como dentro del cañadón se observan rocas provenientes de la erosión del drift glaciario, formando depósitos secundarios. Las morrenas de la última glaciación se extienden desde los cerros Dirección y Aymond hasta el Cabo Vírgenes, en tanto que más al norte se extienden otros depósitos glaciares muy erosionados, probablemente más antiguos, llegando hasta el sur de río Gallegos (Etchichury y Remiro 1967).

\section{DISPONIBILIDAD DE MATERIAS PRIMAS LÍTICAS}

Con el objetivo de evaluar la distribución y disponibilidad de materias primas líticas en la región, se realizaron muestreos de rocas en distintos sectores del espacio, siguiendo la metodología propuesta por Franco y Borrero (1999). La misma consiste principalmente en la elección de lugares a 
muestrear a partir de la información geológica sobre el área, considerando, sin embargo, aquellos lugares que a priori parecerían carecer de las mismas. La recolección de materias primas se realiza recorriendo la fuente potencial de aprovisionamiento, siguiendo una unidad de tiempo establecida y registrándose la cantidad de personas que la efectúan. Se recolectan aquellas rocas aptas para la talla, de las cuales son tomadas muestras. Las variables que se registran son las siguientes: tipo de roca, diámetro máximo, color, calidad para la talla y forma de presentación (guijarro, bloque, filón, etc.). Esta metodología posibilita evaluar el rendimiento relativo de cada fuente. Los resultados obtenidos de los muestreos de rocas llevados a cabo en distintos sectores de Pali Aike y del cañadón Gap, nos permiten comparar la disponibilidad de materias primas líticas en la costa y hacia el interior en el sector sur de la Pcia. de Santa Cruz.

En la mayoría de las investigaciones previas realizadas en Pali Aike y cañadón Gap se ha considerado que el basalto era una de las principales materias primas utilizadas en la confección de los artefactos líticos (Sanguinetti de Bórmida 1976, 1982, 1984; Gómez Otero y Fontanella 1980; Massone e Hidalgo 1981; Nami 1984a, 1984b, 1986; Gómez Otero 1986-87, 1989-90; Prieto 1989-90, 1997; Franco y L'Heureux 2002, entre otros). Sin embargo, los análisis petrográficos y geoquímicos (D'Orazio et al. en preparación) realizados sobre muestras clasificadas macroscópicamente como basalto, han demostrado que se trata de diversos tipos de rocas, tales como vitrófiros dacíticos, lutitas, grauvacas y ftanitas. Dado que no es posible diferenciar estas rocas macroscópicamente (com. pers. Aragón a Franco 2004), han sido denominadas por nosotros Rocas de Grano Fino Oscuras (RGFO) (Charlin 2005). Las mismas se encuentran disponibles en los depósitos glacifluviales del área, aunque en proporción escasa.

\section{El sector de Pali Aike}

En el área de Pali Aike la materia prima predominante en los depósitos secundarios es la dacita, principalmente en coloraciones verdosas y beige, habiéndose recuperado también en bajas proporciones RGFO, calcedonia, jaspe, cuarzo, pórfido dacítico, limolita, tonalita y diabasa.
A pesar de la abundante oferta en Pali Aike de afloramientos primarios de basalto, esta materia prima no ha sido utilizada para la manufactura de los artefactos. Por el contrario, son los depósitos secundarios los que han funcionado como fuentes de aprovisionamiento en este sector del espacio (Gómez Otero 1986-87; Nami 1994). De acuerdo con la localización de los diversos muestreos de rocas realizados, el campo volcánico ha sido dividido en tres áreas: norte (cuenca del río Gallegos), centro (cuenca del río Chico) y sur (frontera con Chile). A continuación se detallan las proporciones de RGFO recuperadas en las diferentes áreas, por ser las rocas más utilizadas en la manufactura de los artefactos, siendo sin embargo su disponibilidad muy escasa. En las terrazas bajas del río Gallegos, los guijarros de RGFO se encuentran disponibles en bajas frecuencias: sobre 10 recolecciones de rocas solamente en cuatro casos han sido registrados nódulos de RGFO (en las proximidades de la cueva de Las Buitreras y de la estancia Palermo Aike). En este último caso se registra una proporción de un nódulo de este tipo de roca cada 25 nódulos recolectados, en tanto que en la zona de Las Buitreras esta relación es de 1:28. Sin embargo, Sanguinetti de Bórmida (1982) señala la abundancia de RGFO en las cotas altas (60 y 90 $\mathrm{m})$ en la margen derecha del río Gallegos.

En el área centro-oriental del campo volcánico esta materia prima sólo ha sido detectada en un caso sobre un total de 162 nódulos recolectados en cuatro muestreos realizados en las márgenes $\mathrm{N}$ y E de la laguna Tres de Enero. Asimismo para esta zona Nami (1986) señala la presencia de "basalto" en la cuenca del río Chico, aunque los muestreos realizados por nosotros en la terraza más baja de este río, en la estancia Markach Aike, no aportaron ningún ejemplar de este tipo de roca. Hacia el oeste de esta zona, dicho investigador refiere la existencia de fuentes localizadas de "basalto" en las lagunas Pali Aike y las del Cerro Mackenzie (Nami 1994). En el sector sur de Pali Aike, sobre la base de 16 muestreos de rocas realizados $(n=394)$ es notable la ausencia de RGFO en los depósitos secundarios.

Entonces, nuestros estudios tienden a señalar el predominio de las dacitas sobre otros tipos de rocas en todos los sectores del campo volcánico, como así también una disminución decreciente de norte a sur en la disponibilidad de RGFO, es decir 


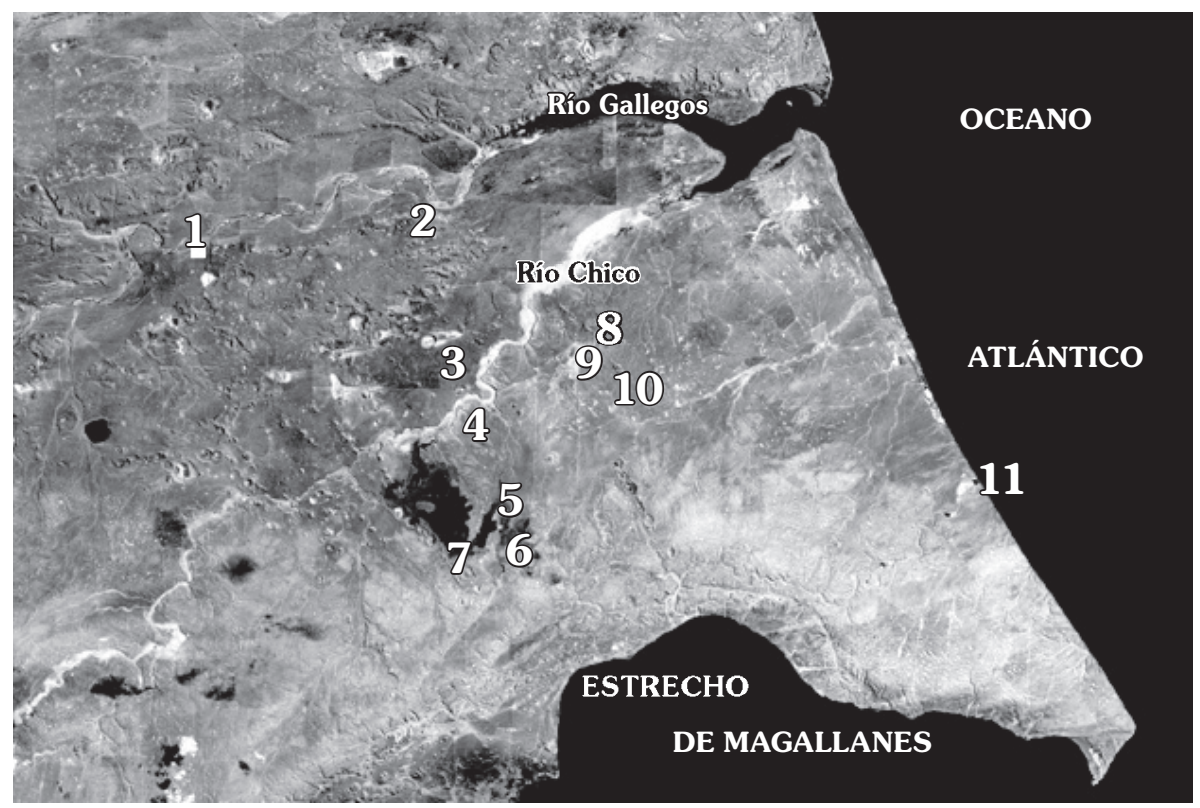

Fig. 1. Procedencia de las muestras de núcleos analizadas

Referencias: 1. Las Buitreras cueva 1; 2. Palermo Aike Estancia y Palermo Aike sitio 2; 3. Markach Aike alero 1;

4. El Volcán cuevas 2 y 4; 5. Laguna Azul; 6. Cueva Orejas de Burro 1; 7. La Olla;

8. Cerro Norte: Aleros Norte 1 y 2; 9. Laguna Tres de Enero; 10. Cueva Cóndor 1; 11. Cañadón Gap.

que la densidad de las mismas disminuye a medida que nos alejamos del río Gallegos. En este punto es importante señalar que además de su escasez, las RGFO se presentan en tamaños pequeños en comparación con otros tipos de rocas, como la dacita. Como ha sido señalado en otro lado (Charlin op. cit.), los guijarros de RGFO presentan tamaños comprendidos entre los 3,5 y $8 \mathrm{~cm}$ de diámetro máximo, predominando aquellos entre los 3,5 y $5 \mathrm{~cm}$. Las dacitas se presentan en tamaños mayores, llegando a un diámetro máximo de $14,5 \mathrm{~cm}$, agrupándose la mayoría de los nódulos entre los 8 y $12 \mathrm{~cm}$.

\section{El sector de cañadón Gap}

En el área del cañadón Gap la disponibilidad de materias primas fue evaluada por la Dra. Franco a partir de muestreos selectivos y recolecciones sistemáticas siguiendo la metodología antes mencionada (Franco MS). Los sectores muestreados comprenden distintos puntos de la costa y del interior. En estos muestreos se observó la existencia de materias primas de buena calidad para la talla, como dacitas, diabasa, gabro y RGFO. Dentro de las dacitas predominan las variedades de color verde y beige (Franco obs. pers.). Estas materias primas provienen de depósitos secundarios, de probable origen glacifluvial. En tanto que en el sector costero de cañadón Gap y probablemente en la costa oeste, del lado chileno, las RGFO están disponibles en forma restringida; la dacita, en cambio, es de distribución más ubicua y abundante en todo el área (Franco obs. pers.). En el sector costero se observó una proporción de 3:25 RGFO en el total de nódulos recolectados en uno de los muestreos realizados y 4:22 en otro, presentando un tamaño entre 4 y $6 \mathrm{~cm}$ de diámetro máximo, semejante a lo observado en Pali Aike. En otros sectores del cañadón, en cambio, no se registraron $\mathrm{RGFO}$ sobre un total de 23 nódulos recolectados.

Es posible afirmar, entonces, el carácter local de la dacita, las RGFO, la diabasa y el gabro. En el área se recuperaron artefactos de calcedonia coloreada y xilópalo en muy bajas proporciones, que no fueron registrados en los muestreos, por lo que se presume que no están disponibles localmente. Las RGFO y la dacita son asimismo las más representadas en todas las categorías artefactuales. Las RGFO son de muy buena calidad para la talla y aparecen en forma de pequeños guijarros. 
Tabla 1. Tipos de materias primas representadas en los núcleos de cañadón Gap y Pali Aike.

\begin{tabular}{|l|c|c|c|c|c|}
\hline \multirow{2}{*}{ Materias Primas } & \multicolumn{2}{|c|}{ Cañadón Gap } & \multicolumn{2}{c|}{ Pali Aike } & \multirow{2}{*}{ Total } \\
\cline { 2 - 5 } & $\mathrm{n}^{\circ}$ & $\%$ & $\mathrm{n}^{\circ}$ & 29 & 53 \\
\hline RGFO & 27 & 71 & 18 & 33 & 26 \\
\hline Dacita & 8 & 21 & 0 & 0 & 0 \\
\hline Diabasa & 2 & 5 & 0 & 6 & 1 \\
\hline Xilópalo & 1 & 3 & 3 & 4 & 3 \\
\hline Calcedonia & 0 & 0 & 2 & 4 & 2 \\
\hline Arenisca & 0 & 0 & 2 & 100 & 2 \\
\hline Pórfido dacítico & 0 & 0 & 54 & & 92 \\
\hline Total & 38 & 100 & & & \\
\hline
\end{tabular}

\section{DESCRIPCIÓN DE LAS MUESTRAS ARTEFACTUALES}

La muestra de núcleos de Pali Aike que se analiza en el presente trabajo, procede de los materiales recuperados en las campañas de los años 2003 y 2004. Se incluyen además muestras previas recolectadas en proyectos dirigidos por la Dra. A. Sanguinetti de Bórmida ${ }^{2}$ (Fig. 1).

La muestra se encuentra constituida por 54 núcleos recuperados en superficie. Los mismos proceden de recolecciones realizadas en sitios arqueológicos, de transectas y de recolecciones controladas, y también se incluyen hallazgos aislados.

Los materiales del área de cañadón Gap que se incluyen en este estudio fueron recogidos durante el año 2001, utilizando transectas sin brazos paralelas al cañadón. Los muestreos (de 100 por 10 metros) fueron discontinuos, siendo recuperados 38 núcleos.

Descripción de los núcleos de Pali Aike

El 89\% de los núcleos se encuentra entero, siendo solamente 6 los casos en los que están fracturados. A pesar de su variabilidad morfológica, todos ellos han sido utilizados para la extracción de lascas (Charlin 2005).

Las materias primas más representadas en la muestra analizada son las RGFO, constituyendo el $53 \%$ del total de la muestra, seguidas por las dacitas, con un $33 \%$ y en porcentajes menores por calcedonia,

2 Han sido incluidas las muestras de núcleos recuperados en superficie en Las Buitreras cueva 1, Palermo Aike Estancia, Palermo Aike sitio 2, Markach Aike alero 1 y El Volcán cueva 2 y cueva 4. arenisca y pórfido dacítico (Tabla 1). Materias primas como jaspe, ópalo, obsidiana (en las variedades de colores gris, verde y negro) y rocas silíceas recuperadas bajo la forma de desechos de talla e instrumentos (en baja frecuencia), no se encuentran representadas entre los núcleos (Charlin op. cit.).

Con respecto a los tipos de núcleos representados, se ha identificado la extracción de lascas con dos y tres elementos. Los núcleos bipolares son los más abundantes con un $31 \%$ del total, seguidos por los núcleos poliédricos con un 26\%. Dentro de esta categoría hemos incluido los núcleos globulosos, pues tanto morfológicamente como considerando la técnica de reducción, constituyen el mismo tipo. La diferencia radica en el grado de explotación entre uno y otro, siendo la variedad globulosa un núcleo poliédrico agotado (Aschero 1983). Los núcleos con lascados aislados representan un $15 \%$ y los núcleos bifaciales un 13\%. En porcentajes menores se registran núcleos discoidales, prismáticos y no diferenciados (Tabla 2).

Como forma base de los núcleos fueron utilizados predominantemente guijarros, conformando el $74 \%$ de la muestra $(n=40)$. Un $13 \%(n=7)$ de las formas base utilizadas se encuentra constituido por artefactos y el $13 \%$ restante es indiferenciado $(n=7)$. Las formas base artefactuales incluyen diferentes tipos de lascas y de instrumentos en proceso de manufactura. En el caso de estos últimos fueron reutilizados artefactos bifaciales con problemas en su formatización (fracturados y/o con abundancia de charnelas), como así también núcleos reclamados (Charlin op. cit.).

Con respecto al tamaño, se ha calculado el volumen de los núcleos enteros $(\mathrm{n}=48)$. El mismo varía entre 5,8 y $395,8 \mathrm{~cm}^{3}$. El volumen promedio es de $83,8 \mathrm{~cm}^{3}$ con un desvío estándar de $96,9 \mathrm{~cm}^{3}$. 
Tabla 2. Tipos de núcleos diferenciados en cañadón Gap y Pali Aike.

\begin{tabular}{|l|c|c|c|c|c|}
\hline \multirow{2}{*}{ Tipo de núcleo } & \multicolumn{2}{|c|}{ Cañadón Gap } & \multicolumn{2}{c|}{ Pali Aike } & \multirow{2}{*}{ Total } \\
\cline { 2 - 5 } & $\mathrm{n}^{\mathbf{0}}$ & $\%$ & $\mathrm{n}^{\mathbf{0}}$ & $\%$ & 2 \\
\hline Prismático & 1 & 3 & 1 & 2 & 17 \\
\hline Bifacial & 10 & 26 & 7 & 13 & 10 \\
\hline Discoidal & 5 & 13 & 5 & 26 & 20 \\
\hline Poliédrico & 6 & 16 & 14 & 31 & 28 \\
\hline Bipolar & 11 & 29 & 17 & 15 & 12 \\
\hline C/ Ls Aislados & 4 & 10 & 8 & 4 & 3 \\
\hline No diferenciado & 1 & 3 & 2 & 100 & 92 \\
\hline Total & 38 & 100 & 54 & & \\
\hline
\end{tabular}

Por otro lado, el $87 \%(n=47)$ de los núcleos presenta corteza. Ha sido registrado el porcentaje de corteza que conservan los núcleos enteros, utilizando categorías segmentadas en cuartos (1 - 25\%, 26 - 50\%, 51 - 75\% y 76 - 100\%). La mayoría de los núcleos presenta porcentajes de corteza comprendidos entre $1-25 \%$ y $26-50 \%$ (Tabla 3) (Charlin op. cit.).

Descripción de los núcleos de cañadón Gap

De los 38 núcleos recuperados en Gap sólo uno se encuentra fragmentado. La categoría tipológica más representada en la muestra son los núcleos bipolares (29\%) y en menor media los bifaciales (36\%) y poliédricos (16\%) (ver Tabla 2). Todos los núcleos fueron utilizados para la extracción de lascas.

Las materias primas más utilizadas son las RGFO (71\%) y en menor medida la dacita (21\%) (ver Tabla 1). Las formas base utilizadas fueron predominantemente guijarros y en sólo tres casos artefactos, dos lascas y un percutor. El volumen medio de los núcleos es de $101 \mathrm{~cm}^{3}$ con un desvío estándar de $139 \mathrm{~cm}^{3}$. Este desvío más grande que la media, se relaciona con la presencia de algunos pocos núcleos de gran tamaño. El 86\% de los núcleos presenta corteza, mientras que sólo un $4 \%$ no presenta ningún remanente de la misma $(n=5)$. $\mathrm{Al}$ igual que en el caso anterior, la mayoría de los núcleos presentan entre 1-25\% de corteza y entre 26-50\% (Tabla 3).

\section{ANÁLISIS Y RESULTADOS}

Para la comparación de ambas muestras se seleccionaron las siguientes variables tecnológicas: Tipo de núcleo (sensu Aschero 1983), volumen, materia prima, presencia de corteza, cantidad y tamaño de las extracciones y formas base. Para el análisis de los datos se utilizaron procedimientos estadísticos paramétricos (F y t test) y no paramétricos (ANOSIM, NPMANOVA y los índices de diversidad de Shannon-Wiener, de dominancia de Simpson y de Homogeneidad -Eveness-).

Para la comparación de los volúmenes y el porcentaje de corteza se dejaron de lado las piezas fragmentadas. En el caso del tamaño de las últimas extracciones, fueron consideradas sólo aquellas que presentaran boca de lascado.

A continuación se presentan los resultados obtenidos en cada una de las variables analizadas.

\section{Materias primas}

En ambas muestras las materias primas con mayor representación son las RGFO y en menor medida la dacita. Sin embargo, las RGFO

Tabla 3. Porcentaje de corteza que conservan los núcleos enteros en cañadón Gap y Pali Aike.

\begin{tabular}{|c|c|c|c|c|c|}
\hline \multirow{2}{*}{ Corteza } & \multicolumn{2}{|c|}{ Cañadón Gap } & \multicolumn{2}{|c|}{ Pali Aike } & \multirow{2}{*}{ Total } \\
\hline & $\mathrm{n}^{\circ}$ & $\%$ & $\mathrm{n}^{\mathrm{o}}$ & $\%$ & \\
\hline $1-25 \%$ & 11 & 34,4 & 23 & 49 & 34 \\
\hline $26-50 \%$ & 14 & 43,7 & 18 & 38 & 32 \\
\hline $51-75 \%$ & 7 & 21,9 & 2 & 4 & 9 \\
\hline $76-100 \%$ & 0 & 0 & 4 & 9 & 4 \\
\hline Total & 32 & 100 & 47 & 100 & 79 \\
\hline
\end{tabular}


Tabla 4. Medidas de diversidad entre las muestras de materias primas.

\begin{tabular}{|l|c|c|c|}
\hline \multicolumn{1}{|c|}{ Índices } & GAP & Pali Aike & Boot $(p=0.05)$ \\
\hline Dominancia & 0.38 & 0.3195 & 0.642 \\
\hline Diversidad & 1.204 & 1.482 & 0.47 \\
\hline Homogeneidad & 0.8336 & 0.8801 & 0.688 \\
\hline
\end{tabular}

se encuentran disponibles en bajas proporciones, mientras que la materia prima de mayor abundancia es la dacita. Para explorar si en ambos conjuntos las materias primas fueron utilizadas de manera semejante se comparó su abundancia y diversidad. Con este fin se calcularon los siguientes índices: el índice de diversidad de Shannon-Wiener, el de dominancia de Simpson y el de homogeneidad basado en el primero. El índice de diversidad es una media de la riqueza de clases en relación al tamaño de la muestra (Krebs 1989). El índice de dominancia de Simpson muestra la medida en que una clase en particular predomina en la muestra, por lo que puede ser utilizado para diferenciar entre conjuntos. Por otro lado el índice de homogeneidad indica el grado en que cada una de las clases tiene una representatividad similar. Por consiguiente, los tres índices están correlacionados; cuanto mayor es el valor obtenido, mayor es la riqueza, dominancia $\mathrm{u}$ homogeneidad de la muestra. Debido a que los índices son estimaciones no paramétricas los niveles de significación para la comparación entre muestras fueron obtenidos mediante remuestreo de 1000 casos (bootstrapping) utilizando el programa PAST (Hammer et al. 2001). La muestra fue estandarizada calculando la raíz cuadrada de los valores originales con el fin de otorgar mayor representatividad a los valores pequeños y homogeneizar la muestra (Krebs op. cit.).

Los resultados muestran valores muy similares (Tabla 4) para los tres índices, lo que sugiere una distribución de las materias primas semejante en ambos conjuntos. Los niveles de significación obtenidos mediante remuestreo no señalan diferencias significativas al 95\% ( $p=0.05)$.

Posteriormente se comparó la abundancia de los diferentes tipos de materias primas a partir del análisis de di-similitudes (ANOSIM) utilizando el índice de abundancia de Bray-Curtis, comúnmente utilizado para comparar comunidades ecológicas. Este test compara la varianza dentro de cada muestra con la variación entre conjuntos, de manera similar al ANOVA (Clarke 1993). El valor R obtenido indica el grado de di-similitud entre dos o más muestras; cuanto mayor es este valor, más grande es la diferencia. Una de las ventajas de la aplicación de este procedimiento es que permite comparar clases con frecuencias muy bajas y valores 0 , así como utilizar medidas de distancia acordes al problema y al tipo de variables utilizadas. El nivel de significación para rechazar la hipótesis nula es obtenido a partir de 5000 replicaciones de los valores originales (Hammer et al. 2001). Para evitar diferencias en la distribución de los datos por el tamaño de las muestras, ambas fueron transformadas a proporciones (Krebs op. cit.).

Los resultados muestran distribuciones semejantes para un nivel de significación del 95\% $(p=0.05)$ (Tabla 5), lo cual es congruente con los valores obtenidos para los índices de diversidad.

$\mathrm{Si}$ bien algunas materias primas utilizadas son diferentes en ambos conjuntos, la tendencia predominante ha sido la utilización mayoritaria de las RGFO y en segundo lugar la dacita. Esto sugiere un comportamiento similar en la selección de recursos líticos, a pesar de la disponibilidad diferencial de RGFO y dacitas registrada en los distintos muestreos.

Tabla 5. Valores del análisis de di-similitudes entre las muestras de materias primas.

\begin{tabular}{|c|c|}
\hline ANOSIM & $\mathrm{R}=-0.08552$ \\
\hline & $\mathrm{p}=0.8514$ \\
\hline
\end{tabular}

Tabla 6. Medidas de diversidad para los tipos de núcleos.

\begin{tabular}{|l|c|c|c|}
\hline \multicolumn{1}{|c|}{ Índices } & GAP & Pali Aike & Boot p(eq) \\
\hline Dominancia & 0.1939 & 0.1667 & 0.595 \\
\hline Diversidad & 1.93 & 1.856 & 0.753 \\
\hline Homogeneidad & 0.9844 & 0.9143 & 0.377 \\
\hline
\end{tabular}


Tabla 7. Valores del análisis de di-similitudes para los tipos de núcleos.

\begin{tabular}{|c|c|}
\hline ANOSIM & R. -0.1244 \\
\hline & $p=0.963$ \\
\hline
\end{tabular}

Tipo de núcleos

Para comparar las técnicas de reducción de núcleos empleadas en ambos espacios, se utilizó el mismo procedimiento estadístico anteriormente descripto. Los resultados obtenidos en ambas muestras son similares, tanto en la variedad como en la abundancia relativa de tipos de núcleos (Tabla 6 y 7).

Los resultados obtenidos sugieren la implementación de estrategias de reducción de núcleos similares en Gap y en Pali Aike, predominando la reducción bipolar.

Volumen de los núcleos

Para comparar el tamaño relativo de los núcleos entre ambas muestras se utilizó el volumen, teniendo en cuenta que los tamaños de los nódulos son semejantes en Pali Aike y Gap, como fue señalado anteriormente. Para ello se consideraron solamente aquellas piezas sin fracturas $(\mathrm{GAP}=37$, Pali Aike $=48$ ). Los valores fueron transformados a logaritmos con lo que la distribución se ajustó a la normalidad, como se contrastó mediante el estadístico de Shapiro-Wilk. (Gap: $\mathrm{W}=0.97, \mathrm{p}=0.67$; Pali Aike $\mathrm{W}=0.96, \mathrm{p}=0.12$ ). Esto permitió aplicar análisis de la igualdad de la varianza y de las medias (test F y t) entre ambos conjuntos (Tabla 8). El test de F señala igualdad de las varianzas en ambos conjuntos, lo que es un requisito para la aplicación del test sobre las medias, más aún al poseer desvíos estándar amplios (ver supra). Los resultados obtenidos muestran varianzas y medias similares a un nivel de confianza del 95\%. Estos resultados sugieren grados de explotación similares en los núcleos de ambos conjuntos.

Tabla 9. Valores del análisis de di-similitudes para los porcentajes de corteza.

\begin{tabular}{|c|c|}
\hline ANOSIM & $\mathrm{R}=-0.194$ \\
\hline & $\mathrm{p}=1$ \\
\hline
\end{tabular}

Tabla 8. Contraste de la media y la varianza en el volumen de los núcleos.

\begin{tabular}{|c|c|c|}
\hline Test & Valor & Nivel de prob \\
\hline $\mathrm{F}$ & 13.586 & 0.32442 \\
\hline $\mathrm{t}$ & 0.11819 & 0.9062 \\
\hline
\end{tabular}

Remanente de corteza

Sobre las piezas enteras, se ha registrado el porcentaje de corteza que conservan tomando intervalos de 25\% (Franco 2002). Aquellos núcleos sin corteza fueron codificados con el número 0 . Se calculó la proporción relativa de cada una de las categorías y se aplicó el ANOSIM (Tabla 9). Los resultados muestran porcentajes similares en ambos conjuntos. Al igual que el resultado anterior, éste apoya la hipótesis de grados de explotación similares.

\section{DISCUSIÓN}

Las comparaciones entre ambos conjuntos señalan la utilización de estrategias similares de explotación de las materias primas líticas. Tanto en el campo volcánico Pali Aike como en el cañadón Gap las materias primas más utilizadas fueron las RGFO, a pesar de su escasa disponibilidad, y en grado menor la dacita. Los análisis realizados sobre los instrumentos recuperados señalan que las RGFO fueron preferidas en la confección de puntas de proyectil y raspadores y en menor medida de cuchillos. La mayor proporción de técnica bipolar aplicada en la reducción de esta roca puede relacionarse con el tamaño pequeño de los nódulos, como ha sido señalado por varios autores (Andrefsky 1998, Mauldin y Amick 1989, Patterson 1987, Shelley 1993, entre otros). Esto puede asimismo explicar las variaciones observadas entre las RGFO y la dacita en cuanto al tamaño de

Tabla 10. Tamaño de las últimas extracciones realizadas en los núcleos.

\begin{tabular}{|l|c|c|}
\hline Últimas extracciones & Largo $(\mathrm{mm})$ & Ancho $(\mathrm{mm})$ \\
\hline Gap & & \\
\hline Dacita & 28,25 & 30 \\
\hline RGFO & 24,25 & 23 \\
\hline Pali Aike & & \\
\hline Dacita & 32,00 & 40,75 \\
\hline RGFO & 24,90 & 19,95 \\
\hline
\end{tabular}


las últimas extracciones y el grado de explotación de los núcleos. Las lascas extraídas de los núcleos son más pequeñas en el caso de las RGFO (Tabla 10) y se registra un mayor porcentaje de núcleos agotados sobre esta materia prima (Gap 99\%, Pali Aike 69\%) que sobre dacita.

\section{CONCLUSIONES}

La evidencia analizada aporta a la discusión sobre el rango de acción de las poblaciones humanas en Patagonia Meridional. Resultados previos obtenidos a partir de distintas líneas de evidencia, como los isótopos estables sobre restos óseos humanos (Borrero et al. 2001; Barberena 2002) y los análisis faunísticos y tecnológicos (Massone 1979; Franco y L'Heureux 2002) entre otros, señalan el uso del espacio costero por poblaciones cazadoras-recolectoras terrestres en el sector sur de Patagonia Meridional. Diversas investigaciones llevadas a cabo en distintos sectores de Pali Aike y en la costa atlántica y del estrecho de Magallanes aportan evidencia para el Holoceno Tardío. En este sentido, Massone e Hidalgo (1981) señalan la explotación de la costa norte del estrecho de Magallanes por grupos cazadores-recolectores tardíos, a partir de la presencia de moluscos entre los restos óseos de guanaco y ñandú recuperados en Pali Aike 2, alero localizado hacia el SW del campo volcánico. Asimismo, sobre la base de sus investigaciones en la costa nororiental del Estrecho de Magallanes, Massone (1979) sostiene que desde el período IV de Bird (ca. 4.500 años AP) hay evidencias de ocupación por parte de cazadores terrestres en la costa meridional del continente, desde el sector nororiental de la península de Brunswick hasta el límite atlántico del estrecho. Estas ocupaciones se extenderían hasta el período histórico. Los sitios datados entre el 3.725 AP y el 360 AP serían el resultado de la acción de estos grupos, que alcanzaban las costas del estrecho aprovechando los recursos litorales disponibles de acuerdo con sus necesidades de subsistencia (Massone op. cit.). Este autor señala la existencia de patrones tecnológicos uniformes en el área, tanto en lo referido a la selección de la materia prima, como a la morfología y tipo de instrumentos utilizados. Estos resultados son concordantes con los obtenidos recientemente para el área de Cabo Vírgenes, en la costa oriental del estrecho. Si bien este espacio habría sido utilizado de manera marginal, habría formado parte del rango regular de acción de los cazadores-recolectores terrestres, al menos hacia el 1.200 AP (Franco y L'Heureux 2002). Por otra parte, la presencia de obsidiana verde -procedente del área del seno de Otway y el mar de Skyring- en sitios costeros y del interior plantea el problema de la forma de adquisición de la misma (Prieto 1989-90; Stern 2000; Morello et al. 2001; Morello et al. 2002; Manzi 2002; Manzi et al. 2003, entre otros).

Entonces, el panorama que surge de estudios previos y de los resultados aquí presentados sugiere que el campo volcánico Pali Aike ha sido explotado por poblaciones de cazadores-recolectores terrestres, que habrían llegado a la costa como parte de su rango de acción habitual, al menos para los últimos 3.700 años radiocarbónicos. Estos grupos habrían utilizado principalmente materias primas como las RGFO y la dacita, obtenidas de forma directa en los depósitos secundarios del área, mientras que habrían obtenido otras, como la obsidiana verde, probablemente por medio del intercambio (Ibíd.).

Los análisis realizados indican la selección que estos grupos realizaron de las RGFO, que a pesar de su escasa disponibilidad fueron muy utilizadas. Esto puede relacionarse con la preferencia de esta materia prima para la confección de puntas de proyectil y con la calidad de la misma (Franco obs. pers.; Nami 1994).

Por último, los resultados obtenidos en este trabajo apoyan la hipótesis de una explotación semejante de las materias primas líticas en el área de Pali Aike y de cañadón Gap. Futuros trabajos apuntarán a relacionar otros aspectos de la tecnología, principalmente con respecto a la intensidad de uso de estos espacios, lo que permitirá ampliar y profundizar los resultados aquí presentados.

\section{AGRADECIMIENTOS}

Los trabajos de investigación fueron realizados en el marco de los subsidios de la Agencia Nacional de Promoción Científica y Técnica PICT 9498, Conicet PIP 2390 y UBACYT F140. Nuestros mayores agradecimientos a Luis Borrero y Nora Franco por la lectura crítica del manuscrito, por sus comentarios y sugerencias. A Ramiro Barberena por la traducción del resumen y su apoyo. 


\section{BIBLIOGRAFÍA}

AGOSTINI, S., D'ORAZIO, M., GONZÁLEZ-FERRAN O., HALLER, M. J., INNOCETTI, E., LAHSEN, A., MANETTI, P., MAZZARINI, F., MAZZUOLI, R. y MEISTER, C. 1999. The Pali Aike Volcanic Field, Southern Patagonia: petrogenesis and geodynamic significance. XIV Congreso Geológico Argentino, Actas II, Salta.

ANDERSON, M.J. 2001. A new method for non-parametric multivariate analysis of variance. Austral Ecology 26: 32-46.

ANDREFSKY, W. 1998. Lithics. Macroscopic approaches to analysis. Cambridge University Press, Cambridge.

ASCHERO, C. 1983. Ensayo para una clasificación morfológica de artefactos líticos aplicada a estudios tipológico-comparativos. Informe CONICET. Revisión 1983. Buenos Aires. Manuscrito.

BARBERENA, R. 2002. Los límites del mar. Isótopos estables en Patagonia Meridional. Colección Tesis de Licenciatura, L. Nacuzzi Ed. Sociedad Argentina de Antropología. Buenos Aires.

BORRERO, L. A. 2001. Modos de interacción entre las poblaciones humanas de la Patagonia Meridional. Proyecto aprobado por la Agencia Nacional de Ciencia y Tecnología. MS. Buenos Aires.

BORRERO, L. A., R. GUICHÓN, R. H. TYKOT, J. KELLY, A. PRIETO y P. CÁRDENAS. 2001. Dieta a partir de isótopos estables en restos óseos humanos de Patagonia austral. Estado actual y perspectivas. Anales del Instituto de la Patagonia, Serie Ciencias Humanas 29: 119-127. Punta Arenas.

CHARLIN, J. 2005. Utilización de materias primas líticas en el campo volcánico Pali Aike (Pcia. Santa Cruz, Argentina). Una primera aproximación a partir del análisis de los núcleos. Werken (5) en prensa. Santiago de Chile.

CLARKE, K. R. 1993. Non-Parametric multivariate analysis of changes in community structure. Australian Journal of Ecology 18:117-143.

CORBELLA, H. 2002. El campo volcánico-tectónico de Pali Aike. En: Geología y recursos naturales de Santa Cruz. Relatorio del XV Congreso Geológico Argentino. El Calafate, 1-18: 285-301. Buenos Aires.

D'ORAZIO, M., S. AGOSTINI, F. MAZZARINI, F. INNOCENTI, P. MANETTI, M. J. ALLER y A. LAHSEN. 2000. The Pali Aike volcanic Field, Patagonia: Slab-Window Magmatism near the Tip of South America. Tectonophysics 321: 407-427.

D'ORAZIO, M., N. FRANCO, E. ARAGÓN y J. CHARLIN. En preparación. Determinación petrográfica y geoquímica de rocas utilizadas en el campo volcánico Pali Aike (Prov. Santa Cruz).

ETCHICHURY M. C. y J. R. REMIRO. 1967. Los sedimentos litorales de la Provincia de Santa Cruz entre Punta Dungeness y Punta Desengaño. Revista del Museo Argentino de Ciencias Naturales "Bernardino Rivadavia", Tomo IV (8): 323-376. Buenos Aires.

FRANCO, N. V. 2002. Estrategias de utilización de recursos líticos en la cuenca superior del río Santa Cruz. Tesis doctoral. Facultad de Filosofía y Letras, Universidad de Buenos Aires.
FRANCO, N. V. y L. A. BORRERO. 1999. Metodología de análisis de la estructura regional de recursos líticos. En: En Los tres Reinos: Prácticas de recolección en el cono Sur de América, C. Aschero, M. Korstanje y P. Vuoto Eds., pp. 27-37, Ediciones Magna Publicaciones, Universidad Nacional de Tucumán. San Miguel de Tucumán.

FRANCO, N. y L. G. L'HEUREUX. 2002. Ocupaciones humanas en el área de Cabo Vírgenes (Pcia. de Santa Cruz, Argentina): El sitio Cabo Vírgenes 6. Anales del Instituto de la Patagonia, Serie Ciencias Humanas 30: 183-201. Punta Arenas.

GÓMEZ OTERO, J. 1986-87. Investigaciones arqueológicas en el alero Potrok-Aike (Provincia de Santa Cruz): Una revisión sobre los períodos IV y V de Bird. Relaciones de la Sociedad Argentina de Antropología XVII/1: 173-198. Buenos Aires.

1989-90. Cazadores tardíos en la zona fronteriza del paralelo $52^{\circ}$ sur. I. El paraje de Juni Aike. Anales del Instituto de la Patagonia, Serie Ciencias Humanas 19: 47-71. Punta Arenas.

GÓMEZ OTERO, J. y M. V. FONTANELLA. 1980. Informe sobre una prospección arqueológica en el extremo sur de la Provincia de Santa Cruz. Karu-Kinka: 91-108. Río Grande.

HAMMER, Ø., HARPER, D.A.T., and P. D. RYAN, 2001. PAST: Paleontological Statistics Software Package for Education and Data Analysis. Palaeontologia Electronica 4 (1): 9. http://palaeo-electronica.org/2001_1/past/ issue1_01.htm

HAMMER Ø. 2005 PAST - Palaeontological Statistics, ver. 1.34. D.A.T. Harper and P.D. Ryan. http://folk.uio. no/ohammer/past

KREBS, C. J. 1989. Ecological Methodology. Harper \& Row, New York.

MANZI, L. 2002. La obsidiana verde como indicador de formas de uso del espacio en Fuego-Patagonia. En: Contra viento y marea. Arqueología de Patagonia. M. T. Civalero, P. M. Fernández y A. G. Guraieb Compiladores, Instituto Nacional de Antropología y Pensamiento Latinoamericano y Sociedad Argentina de Antropología, pp. 117-134. Buenos Aires.

MANZI, L., LANZELOTTI, S., JAIME, J. y M. ORLANDO. 2003. Rangos de acción y territorialidad en el extremo sur de Patagonia. MS. Buenos Aires.

MASSONE, M. 1979. Panorama etnohistórico y arqueológico de la ocupación Tehuelche y Prototehuelche en la costa del Estrecho de Magallanes. Anales del Instituto de la Patagonia, Serie Ciencias Humanas 10: 69-107. Punta Arenas.

MASSONE, M. y E. HIDALGO. 1981. Investigaciones arqueológicas en el alero Pali Aike 2. Anales del Instituto de la Patagonia, Serie Ciencias Humanas 12: 125-140. Punta Arenas.

MAULDIN, R. y D. AMICK. 1989. Investigating patterning in debitage from experimental bifacial core reduction. En: Experiments in Lithic Technology. Ed. D. Amick y R. Mauldin. BAR International Series 528, pp. 65-88.

MORELLO, F., M. SAN ROMÁN, A. PRIETO y CH. STERN. 2001. Nuevos avances para una discusión arqueológica en torno a la obsidiana verde en Patagonia Meridional. 
Anales del Instituto de la Patagonia, Serie Ciencias Humanas 29: 129-148. Punta Arenas.

MORELLO, F., M. SAN ROMÁN Y A. PRIETO. 2002. Obsidiana verde en Fuego-Patagonia: distribución y estrategias tecnológicas. En: Contra viento y marea. Arqueología de Patagonia. M. T. Civalero, P. M. Fernández y A. G. Guraieb Compiladores, Instituto Nacional de Antropología y Pensamiento Latinoamericano y Sociedad Argentina de Antropología, pp. 149-166. Buenos Aires.

NAMI, H. 1984a. Análisis tipológico de los instrumentos provenientes del sitio "El Volcán" C. 4. Cuenca del Río Chico, Provincia de Santa Cruz. PREP: Informes de Investigación 1: 55-81. Buenos Aires.

1984b. Algunas observaciones sobre la manufactura de las puntas de proyectil de El Volcán. PREP: Informes de Investigación 1:85-107. Buenos Aires.

1986. Experimentos para el estudio de la tecnología bifacial de las ocupaciones tardías en el extremo sur de Patagonia Continental. PREP: Informes de Investigación 5: 1-120. Buenos Aires.

1994. Paleoindio, cazadores-recolectores y tecnología lítica en el extremo sur de Sudamérica continental. En: Arqueología de Cazadores-Recolectores. Límites, Casos y Aperturas. J. L. Lanata y L. A. Borrero compiladores. Arqueología Contemporánea 5, Edición Especial, pp.89-103. Buenos Aires.

PATTERSON, L. 1987. Amorphous cores and utilized flakes: A commentary. Lithic Technology, vol. 16 (2-3): 51-64.

PRIETO, A. 1989-90. Cazadores tardíos en la zona fronteriza del paralelo $52^{\circ}$ sur. II. El alero Peggy Bird. Anales del
Instituto de la Patagonia, Serie Ciencias Humanas 19. 73-85. Punta Arenas.

1997. Algunos resultados de los trabajos arqueológicos en Juni Aike 2. Anales del Instituto de la Patagonia, Serie Ciencias Humanas 25: 137-146. Punta Arenas.

SANGUINETTI DE BÓRMIDA, A. 1976. Excavaciones prehistóricas en la cueva de Las Buitreras, Santa Cruz, Argentina. Relaciones de la Sociedad Argentina de Antropología X: 271-292. Buenos Aires.

1982. Introducción a la prehistoria de la Patagonia Argentina. Tesis doctoral. Facultad de Filosofía y Letras. Universidad de Buenos Aires. MS. Buenos Aires.

1984. Noticias sobre el sitio "El Volcán", su relación con el poblamiento tardío de las cuencas de los ríos Gallego y Chico (Provincia de Santa Cruz, Argentina). PREP: Informes de Investigación 1: 5-34. Buenos Aires.

SHELLEY, P. 1993. A geoarchaeological approach to the analysis of secondary lithic deposits. Geoarchaeology: An International Journal, vol. 8 (1): 59-72.

SKEWES, M. 1978. Geología, petrología, quimismo y origen de los volcanes del área de Pali-Aike, Magallanes, Chile. Anales del Instituto de la Patagonia 9: 95-106. Punta Arenas.

STERN, CH. 2000. Sources of obsidian artefacts from the Pali Aike, Fell's Cave and Cañadón La Leona archaeological sites in southernmost Patagonia. En: Desde el país de los gigantes. Perspectivas arqueológicas en Patagonia, tomo II, pp. 43-55. Río Gallegos, UNPA. 
